\title{
Comportamiento ante la transmisión del VIH/sida en adolescentes $y$ jóvenes en universidades privadas de Villavicencio (Meta, Colombia)'
}

\author{
Emilce Salamanca Ramos²
}

Esperanza Romero González ${ }^{3}$

doi:10.11144/Javeriana.ie19-2.ctva

Cómo citar: Salamanca Ramos E, Romero González E. Comportamiento ante la transmisión del VIH/sida en adolescentes y jóvenes en universidades privadas de Villavicencio (Meta, Colombia). Investig Enferm Imagen Desarr. 2017;19(2):53-67. http://dx.doi. org/10.11144/Javeriana.ie19-2.ctva

1. Artículo original de investigación. Fecha de recepción: 26 de junio de 2015. Fecha de aceptación: 26 de abril de 2016.

2. Enfermera. Magíster en Enfermería. Profesora de la Facultad Ciencias de la Salud Universidad de los Llanos, Villavicencio, Colombia. Correo electrónico: esalamanaca@unillanos.eduo.co

3. Enfermera. Magister en Enfermería. Profesora de la Facultad Ciencias de la Salud Universidad de los Llanos, Villavicencio, Colombia. Correo electrónico: eromero@unillanos.edu.co 


\section{Resumen}

El Plan Nacional de Respuesta ante la Infección del VIH/Sida en Colombia hace hincapié en fomentar conocimientos, actitudes, comportamientos y prácticas para el desarrollo de una sexualidad autónoma, responsable y placentera. Objetivo: Identificar comportamientos de riesgo para la trasmisión del VIH/sida en adolescentes y jóvenes de las universidades privadas del municipio de Villavicencio. Método: Estudio descriptivo. Diseño de muestreo probabilístico multietápico. Población: 10.324 estudiantes-IP 2014 ( $\mathrm{n}=357$ ). Error: 1\%; nivel de confianza: 95\%. Se utilizó la encuesta Conocimientos, prácticas y asesoría en prueba voluntaria para VIH en jóvenes de 15 a 24 años, y la información se procesó con el programa SPSS v. 14. Resultados: El 95\% de los estudiantes tiene conocimientos sobre el VIH/sida. La principal fuente de información son los programas de televisión (28,6\%). Para el 51,6\% su primera relación sexual ocurrió antes de los 16 años de edad; para el 32,5\%, entre 17 y 19 años. E1 31,9\% no utilizó condón en la primera relación sexual. E1 86,6\% considera que el sida se puede prevenir usando condón. De los encuestados, han tenido una pareja sexual en el último año, el 35,5\%; entre 2 y 4 parejas sexuales, el 37,2\%; más de 5, el 13,4\%. E1 31,1\% no utilizó condón en su última relación. Conclusiones: El estudio indica que las prácticas sexuales de riesgo de la población estudiada es el inicio temprano de las relaciones sexuales, hay un predominio significativo de no uso de condón, a pesar de identificarlo como una forma de prevención de la infección por VIH. Es importante desarrollar estrategias educativas para la promoción de la salud en contexto universitario que fomenten estilos de vida saludables.

Palabras clave: factores de riesgo; VIH; sida; comportamiento; adolescentes/jóvenes

\section{Behavior in Face of the Transmission of HIV / AIDS in Adolescents and Young People in Private Universities in Villavicencio (Meta, Colombia)}

\section{Abstract}

The National Response Framework for HIV / AIDS infection in Colombia (In Spanish, Plan Nacional de Respuesta ante la Infección del VIH/Sida) emphasizes the promotion of knowledge, attitudes, behaviors and practices for the development of an autonomous, responsible and pleasurable sexuality. Objective: To identify risk behaviors for transmission of HIV / AIDS in adolescents and young people from private universities in the municipality of Villavicencio. Method: A descriptive study. Multistage probability sampling design. Population: 10,324 students-IP 2014 ( $\mathrm{n}=357$ ). Error: 1\%; confidence level: $95 \%$. The survey Conocimientos was used for gathering information as well as, practices and counseling in voluntary testing for HIV in young people aged 15 to 24 , the program that was used to process information was SPSS v. 14. Results: 95\% of students have knowledge about HIV / AIDS. The main source of information is television programs (28.6\%). 51.6\% had their first intercourse before age 16;32.5\% between 17 and 19 years. 31.9\% did not use a condom at first intercourse. $86.6 \%$ believe that AIDS can be prevented by using condoms. From all respondents, $35.5 \%$ have had a sexual partner in the last year, 37.2\% between 2 and 4 sexual partners, 13.4\% more than 5. $31.1 \%$ did not use condoms in their last relationship. Conclusions: The study suggests that the risky sexual practices from the population studied is the early onset of sexual relations, there is also a significant predominance of non-condom use despite of the fact that they identify it as a way of preventing HIV infection. It is important to develop educational strategies for health promotion in university context that encourage healthy lifestyles. 


\section{Comportamento ante a transmissão de VIH/Sida em adolescentes e jovens em universidades privadas de Villavicencio (Meta, Colômbia)}

\section{Resumo}

O Plan Nacional de Respuesta ante la Infección del VIH/Sida en Colombia (Plano Nacional de Resposta ante a Infeção do VIH/Sida na Colômbia) enfatiza o fomento de conhecimentos, atitudes, comportamentos e práticas para o desenvolvimento de uma sexualidade autónoma, responsável e prazerosa. Objetivo: Identificar comportamentos de risco para a transmissão do VIH/Sida em adolescentes e jovens das universidades privadas do município de Villavicencio. Método: Estudo descritivo. Design de amostragem probabilístico em múltiplos estágios. População: 10.324 estudantes-IP 2014 (n = 357). Erro: $1 \%$; nivel de confianza: $95 \%$. Utilizou-se o inquérito Conhecimentos, práticas e aconselhamento em testes voluntários para VIH em jovens de 15 a 24 anos, e as informações foram processadas com o programa SPSS v. 14. Resultados: $95 \%$ dos estudantes têm conhecimento sobre VIH/Sida. A principal fonte de informação são os programas de televisão $(28,6 \%)$. Para $51,6 \%$, o primeiro relacionamento sexual aconteceu antes dos 16 anos de idade; para 32,5\%, entre 17 e 19 anos. 31,9\% não utilizou camisinha no primeiro relacionamento sexual. $86,6 \%$ acredita que é possivel prevenir a Sida usando camisinha. Dos inqueridos, 35,5\% tiveram apenas um parceiro sexual no último ano; $37,2 \%$ entre 2 e 4 parceiros sexuais e $13,4 \%$ mais do que 5 . $31,1 \%$ não utilizou camisinha no último relacionamento. Conclusões: O estudo sugere que as práticas sexuais de risco da população estudada estão no inicio precoce de relacionamentos sexuais e há predomínio significativo de não uso de camisinha, embora identificá-lo como forma de prevenção da infecção pelo VIH. Resulta importante desenvolver estratégias educativas de promoção de saúde no contexto discente para fomentarem estilos de vida saudáveis.

Palavras-chave: fatores de risco; VIH; sida; comportamento; adolescentes/jovens 


\section{Introducción}

La epidemia de VIH/sida ha afectado todas las esferas estructurales de las sociedades y ha evidenciado la necesidad de comprender la infección como un fenómeno social (1). El VIH continúa siendo uno de los agentes infecciosos más mortíferos del mundo. Desde el comienzo de la epidemia, aproximadamente 78 millones (71-87 millones) de personas contrajeron la infección por VIH y 39 millones (35-43 millones) de personas han fallecido a causa de enfermedades relacionadas con el VIH. En América Latina, en 2013, en promedio 1,6 millones de personas vivía con el VIH; pero en la región se produjeron aproximadamente 94.000 nuevas infecciones por VIH. Sin embargo, en el periodo 2005-2013 las nuevas infecciones por VIH descendieron un 3\%. En 2013, en promedio 47.000 personas murieron por causas relacionadas con el VIH/sida, y el número de muertes asociado con la enfermedad experimentó un descenso del 31\% en el periodo 2005-2013 (2).

En Colombia, la población más afectada por la epidemia es el grupo entre los 15 y los 34 años de edad, con el 56,5\% (30.796) de los casos notificados por VIH/sida y muerte; seguido de los mayores de 45 años, con el 15,2\% de la participación (8282 casos), en el 2011 (3). Para el 2012, el grupo de edad con el mayor número de casos notificados de VIH/sida fue el de 25 a 29 años de edad, con cerca del $20 \%$ de los casos. Le sigue el grupo de edad de las personas entre 20 y 24 años, y, en tercer lugar, el de 30 a 34 años. Estos tres grupos de edad abarcan una buena proporción de la población económicamente productiva del país (4). Según datos de la Secretaría de Salud del Meta, entre 2013 y 2015 se notificaron 405 casos, de los cuales 277 corresponde a Villavicencio, su capital.

Para 2012 se notificaron 8197 muertes por VIH/sida; para 2013, 8306 casos; para 2014, 9700 casos, y para 2015, 11.606 casos, lo que muestra un aumento entre 2014 y 2015 del 14,9\% (1513 casos) (5).

La infección del VIH/sida sigue vinculada a los comportamientos sexuales. La población más vulnerable son los adolescentes (6) y los jóvenes $(7,8)$, por sus características psicosociales y físicas. Estas etapas representan cambios en el inicio de las relaciones sociales, afianzamiento de actitudes, valores, creencias y pensamientos que inciden en los comportamientos saludables o de riesgo. Los comportamientos ante el VIH/sida que reporta con mayor frecuencia la literatura científica son: inicio de su primera relación sexual sin protección, relaciones sexuales con múltiples parejas, no usar efectiva y consistentemente el condón (9) en todas sus relaciones sexuales, conducta que facilita la adquisición de infecciones y embarazos no deseados, lo que se convierte en un reto para la salud pública y para la misma sociedad en las últimas décadas.

En la actualidad, el número de estudiantes menores de 18 años de edad que ingresa a las universidades aumenta progresivamente. Cada vez es menor la edad de ingreso y son pocos los estudiantes que cuentan con las habilidades necesarias para asumir los compromisos y los riesgos del entorno universitario, así como para asumir nuevas experiencias personales y sociales. La universidad trae nuevos retos y experiencias para los jóvenes, como la presión social de tener nuevas experiencias sexuales, el manejo del 
tiempo fuera del control de los padres, entre otros. De igual forma, algunos estudiantes presentan crisis de identidad que puede ocasionar problemas en la sexualidad, consumo de sustancias psicoactivas, trastornos alimentarios, depresión o suicidio, aunque la interacción cultural y el ambiente académico intenten fomentar un desarrollo saludable (9).

El comienzo de la vida universitaria en los adolescentes y jóvenes trae consigo diferentes cambios en el desarrollo de costumbres y hábitos de riesgo, entre los que destacan el alcoholismo, el tabaquismo, la drogadicción y las prácticas sexuales de riesgo (10). En Colombia, la política de salud sexual y reproductiva (4) aún no ha incidido sustancialmente en la problemática de la salud sexual y reproductiva de los adolescentes y jóvenes universitarios. Estudios realizados en jóvenes universitarios de la ciudad de Villavicencio indican que las edades promedio de ingreso están entre los 16 y los 18 años, y en su mayoría son solteros $(11,12)$. Es importante que la universidad reconozca que los jóvenes y los adolescentes que ingresan tienen unas características especiales y están susceptibles a los cambios que se presentan en el entorno que los rodea. Generalmente, se conciben y se tratan como adultos, pero se excluyen condiciones respecto al ciclo vital en que se encuentran, que los hace más vulnerables a comportamientos de riesgo que afectan la salud de los estudiantes.

Un estudio sobre los hábitos de salud de la población universitaria indica (10) que, por ser una población joven, esta no dimensiona los problemas de salud que aparecen en edades posteriores. De ahí que el objetivo de la investigación haya sido identificar los comportamientos de riesgo para la trasmisión del VIH/sida en los adolescentes y jóvenes de las universidades privadas del municipio de Villavicencio, con el propósito de revisar algunos componentes de la enfermedad, fortalecer el currículo a través de líneas de profundización, en la formación de profesionales en la salud, y generar estrategias de promoción de la salud en los programas de bienestar universitarios en la universidades de la región.

\section{Método}

La investigación tuvo un enfoque cuantitativo, descriptivo y transversal. La población fue de 10.324 estudiantes, y para el cálculo de la muestra se utilizó un muestreo probabilístico multietápico, que tuvo en cuenta el porcentaje de incidencia 1,6 de VIH/sida en el municipio de Villavicencio para el 2013. Así mismo, se consideró la distribución por carrera, semestre y género para 357 estudiantes. El nivel de confianza fue del 95\%, con un porcentaje de error del $1 \%$ y máxima variabilidad, por no existir antecedentes en el municipio sobre la investigación. Los criterios de inclusión fueron los estudiantes activos de universidades privadas de Villavicencio, tener entre 15 y 24 años de edad, aceptar el asentimiento y el consentimiento informado. Se utilizó la encuesta "Conocimientos, prácticas y asesoría en prueba voluntaria para VIH en jóvenes de 15 a 24 años", de la Guía de prevención VIH/sida en jóvenes en contexto de vulnerabilidad, avalado por el Ministerio de la Protección Social y el Fondo de Población de las Naciones Unidas-Colombia 2011 (1). La encuesta la utilizan los programas de salud dirigidos a los adolescentes y 
jóvenes en el municipio de Villavicencio, e incluyó información sociodemográfica, preguntas para evaluar los conocimientos sobre VIH/sida, de actitudes frente a la infección, sobre las prácticas protectoras frente a esta entidad, incluida la realización de la prueba para detectar el VIH.

Los datos se procesaron con el programa SPSS ${ }^{\circledR}$ versión 14 , que permitió utilizar estadística descriptiva, como medidas de tendencia central y dispersión. En cumplimiento con lo establecido en los requerimientos éticos, se tuvo presente lo expuesto en la Resolución 008430 de 1993 del Ministerio de Salud de Colombia y la autorización por parte de las directivas de las instituciones educativas.

\section{Resultados}

La tabla 1 indica algunas características sociodemográficas de los estudiantes, por ejemplo, el rango de edades que más predomina está entre los 18 y los 24 años; hay igual proporción de hombres y mujeres; la mayoría de la población es soltera; los estratos que más predominan son el 2 y el 3. Así mismo, la tipología de familia de mayor predominio es la nuclear, seguida de la extensa.

TABLA 1. Características sociodemográficas de estudiantes universidades privadas de la ciudad de Villavicencio, Colombia, 2014 ( $\mathrm{n}=357)$

\begin{tabular}{|c|c|c|c|}
\hline \multicolumn{2}{|c|}{ Caracteristica } & \multirow{2}{*}{$\mathbf{n}=\mathbf{3 5 7}$} & \multirow{2}{*}{$\begin{array}{ll}\% & \\
& 1,1 \\
\end{array}$} \\
\hline \multirow{5}{*}{ Edad (años) } & 15 a 16 & & \\
\hline & 17 a 18 & 57 & 16,0 \\
\hline & 19 a 20 & 104 & 29,1 \\
\hline & 21 a 22 & 76 & 21,3 \\
\hline & 23 a 24 & 116 & 32,5 \\
\hline \multirow{2}{*}{ Sexo } & Hombre & 185 & 51,8 \\
\hline & Mujer & 172 & 48,2 \\
\hline \multirow{5}{*}{ Tipología familiar } & Nuclear & 239 & 67,0 \\
\hline & Extensa & 40 & 11,2 \\
\hline & Monoparental & 7 & 2,0 \\
\hline & Familia ampliada & 3 & 0,8 \\
\hline & Sin núcleo familiar & 68 & 19,0 \\
\hline \multirow{5}{*}{ Estrato socioeconómico } & Uno & 22 & 6,2 \\
\hline & Dos & 85 & 23,8 \\
\hline & Tres & 213 & 59,7 \\
\hline & Cuatro & 33 & 9,2 \\
\hline & Cinco & 4 & 1,1 \\
\hline \multirow{5}{*}{ Estado civil } & Soltero & 305 & 85,4 \\
\hline & Casado & 8 & 2,2 \\
\hline & Comprometido & 14 & 3,9 \\
\hline & Unión libre & 28 & 7,8 \\
\hline & Divorciado & 2 & 0,6 \\
\hline
\end{tabular}

Fuente: resultados de la investigación 
Los resultados indican que el $95 \%$ de los estudiantes ha oído hablar sobre el VIH/sida: la principal fuente de información está constituida por los programas de televisión (28,6\%), seguido del establecimiento educativo universitario $(22,4 \%)$ y campañas de salud $(15,1 \%)$ (tabla 2$)$.

TABla 2. Fuentes de información sobre $\mathrm{VIH} /$ sida de estudiantes universidades privadas de la ciudad de Villavicencio, Colombia, 2014 ( $\mathrm{n}=357)$

\begin{tabular}{|l|r|r|}
\hline \multicolumn{1}{|c|}{ Fuente } & n & \multicolumn{2}{c|}{$\%$} \\
\hline Televisión & 102 & 28,6 \\
\hline Radio/prensa & 11 & 3,1 \\
\hline Familia & 25 & 7,0 \\
\hline Institución de salud & 24 & 6,7 \\
\hline Amigos & 12 & 3,4 \\
\hline Establecimiento educativo & 80 & 22,4 \\
\hline Campañas de salud & 54 & 15,1 \\
\hline Internet & 33 & 9,2 \\
\hline No responde & 16 & 4,5 \\
\hline Total & 357 & 100,0 \\
\hline
\end{tabular}

Fuente: resultados de la investigación

Los participantes (tabla 3) consideran: el VIH/sida se puede prevenir usando condón (86.6\%), se transmite por la picadura de un zancudo o mosquito $(12,4 \%)$, se puede prevenir teniendo una sola pareja sexual (40\%).

Tabla 3. Conocimiento sobre el VIH/sida y sexo en estudiantes universidades privadas de la ciudad de Villavicencio, Colombia, 2014 ( $\mathrm{n}=357)$

\begin{tabular}{|c|c|c|c|c|c|c|c|}
\hline \multirow{3}{*}{ Pregunta } & \multicolumn{5}{|c|}{ Sexo } & \multirow{2}{*}{\multicolumn{2}{|c|}{ Total }} \\
\hline & \multicolumn{2}{|c|}{ Hombre } & \multicolumn{2}{|c|}{ Mujer } & \multirow[b]{2}{*}{$\mathbf{n}$} & & \\
\hline & $\mathbf{n}$ & $\%$ & $\mathbf{n}$ & $\%$ & & $\mathbf{n}$ & $\%$ \\
\hline \multirow{3}{*}{$\begin{array}{l}\text { El VIH se puede } \\
\text { prevenir teniendo una } \\
\text { sola pareja sexual }\end{array}$} & Sî & 69 & 19,3 & 74 & 20,7 & 143 & 40 \\
\hline & No & 97 & 27,2 & 80 & 22,4 & 177 & 49,6 \\
\hline & NS/NR & 19 & 5,3 & 18 & 5,1 & 37 & 10,4 \\
\hline \multirow{3}{*}{$\begin{array}{l}\text { El VIH se puede } \\
\text { prevenir usando el } \\
\text { condón }\end{array}$} & Sí & 163 & 45,7 & 146 & 40,9 & 309 & 86,6 \\
\hline & No & 12 & 3,4 & 10 & 2,8 & 22 & 6,2 \\
\hline & NS/NR & 10 & 2,8 & 16 & 4,5 & 26 & 7,3 \\
\hline \multirow{3}{*}{$\begin{array}{l}\text { El VIH se puede } \\
\text { transmitir por la } \\
\text { picadura de un } \\
\text { zancudo o mosquito }\end{array}$} & Sí & 22 & 6,2 & 22 & 6,2 & 44 & 12,4 \\
\hline & No & 126 & 35,3 & 128 & 35,8 & 254 & 71,1 \\
\hline & NS/NR & 37 & 10,3 & 22 & 6,2 & 59 & 16,5 \\
\hline
\end{tabular}




\begin{tabular}{|l|l|r|r|r|r|r|r|}
\hline \multirow{2}{*}{$\begin{array}{l}\text { El VIH se puede } \\
\text { transmitir por } \\
\text { compartir comida con } \\
\text { una persona infectada }\end{array}$} & Sí & 12 & 3,4 & 20 & 5,6 & 32 & 9 \\
\cline { 2 - 8 } & No & 134 & 37,5 & 127 & 35,6 & 261 & 73,1 \\
\hline \multirow{2}{*}{$\begin{array}{l}\text { Cree que una } \\
\text { persona que se ve } \\
\text { sana puede estar } \\
\text { infectada con VIH }\end{array}$} & Sín & 151 & 42,3 & 141 & 39,5 & 292 & 81,8 \\
\cline { 2 - 8 } & No & 15 & 4,2 & 16 & 4,5 & 31 & 8,7 \\
\cline { 2 - 8 } & NS/NR & 19 & 5,3 & 15 & 4,2 & 34 & 9,5 \\
\hline
\end{tabular}

Fuente: resultados de la investigación

La tabla 4 indica los comportamientos presentes en los universitarios. Una minoría no ha tenido relaciones sexuales $(8,1 \%)$, la primera relación sexual fue antes de los 16 años (51,6\%) y el 31,9\% no utilizó condón. De este grupo, ha tenido relaciones sexuales con una persona en el último año el 35,5\%; entre dos y cuatro personas, el 37,2\%, y con más de cinco, el $13,5 \%$. El grupo de estudiantes entre 21 y 24 reporta el mayor número de parejas sexuales en el último año.

TABLA 4. Comportamientos sexuales en los universitarios de universidades privadas de la ciudad de Villavicencio, Colombia, $2014(\mathrm{n}=357)$

\begin{tabular}{|l|r|r|r|r|r|r|r|r|}
\hline & \multicolumn{7}{|c|}{ Edad agrupada } & \multicolumn{2}{c|}{ Total } \\
\cline { 2 - 9 } & $\mathbf{1 5}$ a 16 & $\mathbf{1 7}$ a 18 & $\mathbf{1 9}$ a 20 & $\mathbf{2 1}$ a 22 & $\mathbf{2 3}$ a 24 & $\mathbf{n}=\mathbf{3 5 7}$ & \multicolumn{1}{c|}{$\%$} \\
\hline Persona con la que tuvo su primera relación sexual \\
\hline Hombre & 0 & 21 & 53 & 38 & 55 & 167 & 46,8 \\
\hline Mujer & 2 & 19 & 42 & 37 & 57 & 157 & 44,0 \\
\hline No ha tenido & 2 & 13 & 8 & 2 & 4 & 29 & 8,1 \\
\hline No responde & 0 & 3 & 1 & 0 & 0 & 4 & 1,1 \\
\hline \multicolumn{7}{|c|}{ Uso de condón en esa primera relación sexual } \\
\hline Sí & 1 & 34 & 69 & 46 & 64 & 214 & 60,0 \\
\hline No & 1 & 10 & 28 & 28 & 48 & 114 & 31,9 \\
\hline No ha tenido & 2 & 13 & 8 & 2 & 4 & 29 & 8,1 \\
\hline \multicolumn{7}{|c|}{ Con cuántas personas ha tenido relaciones sexuales penetrativas } \\
\hline anal, oral o vaginal en el último año & \\
\hline Una & 1 & 15 & 35 & 27 & 49 & 127 & 35,6 \\
\hline Dos & 0 & 7 & 18 & 11 & 24 & 60 & 16,8 \\
\hline Tres & 1 & 8 & 20 & 13 & 12 & 54 & 15,1 \\
\hline Cuatro & 0 & 2 & 7 & 7 & 3 & 19 & 5,3 \\
\hline Más de cinco & 0 & 5 & 12 & 12 & 19 & 48 & 13,5 \\
\hline No aplica & 2 & 13 & 8 & 2 & 4 & 29 & 8,1 \\
\hline Ninguna & 0 & 2 & 1 & 1 & 0 & 4 & 1,1 \\
\hline No responde & 0 & 6 & 2 & 3 & 5 & 16 & 4,5 \\
\hline
\end{tabular}

Fuente: resultados de la investigación

La tabla 5 indica que el 31,1\% de los encuestados no utilizó condón en su última relación sexual. Entre las razones de no uso está para el 9,2\%, 
porque confian en la pareja (los que más confian son los hombres). El lugar de más confianza o seguridad a la hora de conseguir o comprar condones en la farmacia/drogueria, seguido del supermercado. En igual proporción de sexos han tenido relaciones sin condón bajo efectos del alcohol (25,5\%), el $4,1 \%$ ha recibido dinero a cambio de tener relaciones sexuales, y este comportamiento predomina más en hombres; de ellos, utilizaron condón el 2,8\%.

TaBla 5. Comportamientos de riesgo en estudiantes universidades privadas de la ciudad de Villavicencio, Colombia, 2014

\begin{tabular}{|c|c|c|c|c|}
\hline & Hombre & Mujer & $\mathbf{n}=\mathbf{3 5 7}$ & $\%$ \\
\hline \multicolumn{5}{|c|}{ ¿Utilizaron condón? } \\
\hline Sí & 110 & 103 & 213 & 59,7 \\
\hline No & 60 & 51 & 111 & 31,1 \\
\hline No ha tenido & 14 & 15 & 29 & 8,1 \\
\hline No responde & 1 & 3 & 4 & 1,1 \\
\hline \multicolumn{5}{|c|}{ Si no utilizó condón, la principal razón fue: } \\
\hline A la pareja no le gusta & 13 & 8 & 21 & 5,9 \\
\hline Cree que disminuye lo que siente & 9 & 8 & 17 & 4,8 \\
\hline La pareja estaba bajo efectos del alcohol & 4 & 1 & 5 & 1,4 \\
\hline No lo vio necesario & 7 & 13 & 20 & 5,6 \\
\hline Confía en la fidelidad de la pareja & 18 & 15 & 33 & 9,2 \\
\hline Estaba muy excitado(a) & 7 & 5 & 12 & 3,4 \\
\hline Otros & 1 & 2 & 3 & 0,8 \\
\hline No aplica & 123 & 117 & 240 & 67,2 \\
\hline No sabe/no responde & 3 & 3 & 6 & 1,7 \\
\hline
\end{tabular}

Fuente: resultados de la investigación

En el último año, el 17,4\% de los estudiantes encuestados se ha realizado la prueba para detectar VIH/sida. Los motivos fueron: iniciativa propia $(12 \%)$ o solicitud de trabajo (2\%). Se encontró una relación entre conocimiento del VIH/sida y la realización de la prueba: el 59,4\% conoce dónde realizarse la prueba de $\mathrm{VIH} /$ sida. El 17,4\% conoció los resultados de la prueba la última vez que se la realizó; el 13,7\% recibió algún tipo de asesoría o consejería antes de tomarse el examen, y un 11,8\% cuando le entregaron los resultados de la prueba. El lugar que más frecuentan para la prueba es el centro de salud (7,8\%), seguido de las clínicas (5\%). Finalmente, el 17,6\% de los estudiantes manifiesta haber recibido condones en el servicio de salud al que pertenecen. E1 96,6\% refiere no haber contraído alguna infección de trasmisión sexual, y los que las presentaron (en un bajo porcentaje) fueron herpes y VIH positivo.

\section{Discusión}

Las características sociodemográficas de la población estudiada corresponden a una muestra de 357 estudiantes de universidades privadas del municipio de Villavicencio. En cuanto a las edades y sexo de los participantes, son 
similares a otras investigaciones relacionadas con universitarios $(8,13,14)$. Los participantes se encuentran en una etapa de la vida (6) en la cual ocurren los cambios más trascendentales, puesto que allí se consolidan o se adoptan estilos de vida que generarán un impacto positivo o negativo en su estado físico, emocional, familiar y social (13) que podrán mantener (15) en edades posteriores. Los comportamientos saludables de los jóvenes, incluida su vida sexual, están condicionados por muchos factores, entre ellos el abandono del domicilio familiar, la interacción con personal de diferentes contextos y sitios de origen, las condiciones económicas, la red de apoyo familiar y las condiciones mismas que la universidad ofrece para la promoción de la salud de los jóvenes.

En lo relacionado con los conocimientos y algunas creencias del VIH/ sida, la mayoría de la población ha oído hablar del tema. Las principales fuentes de información han sido programas de televisión, seguido de establecimiento educativo y campañas de salud. Sin embargo, un número importante no ha participado en actividades de información o educación sobre $\mathrm{VIH} /$ sida $(73,9 \%)$, lo que hace que el grupo de participantes tenga pocos conocimientos o conocimientos inadecuados sobre sexualidad (9), y la forma de prevenir las enfermedades de transmisión sexual, lo cual aumenta la vulnerabilidad frente a la infección por VIH.

No obstante, los participantes consideran que el VIH/sida se puede prevenir usando condón $(86,6 \%)$ y teniendo una sola pareja sexual estable $(40 \%)$. Ello es contradictorio a sus prácticas sexuales, por el número de parejas sexuales que han tenido en el último año. Los conocimientos y la percepción de riesgo entre los adolescentes y jóvenes universitarios sobre VIH/sida se han estudiado en muchas oportunidades, pero se considera que el tener conocimientos sobre la enfermedad es insuficiente para generar conductas preventivas frente a la infección (16). En otros casos, lo asocian como primera línea de defensa frente al riesgo de infección por VIH/sida, siempre y cuando se brinden tales conocimientos bajo una perspectiva holística y que integre la red relacional del adolescente (17). Los conocimientos y comportamientos sobre el VIH/ sida condicionan las prácticas de conductas sexuales saludables, siendo un componente mediador de los factores de riesgo en los adolescentes y jóvenes.

Se encontraron desconocimientos en algunos participantes, como considerar que el VIH/sida se puede transmitir por la picadura de un zancudo o mosquito, creer que se puede transmitir por compartir comida con una persona infectada y considerar que no se puede prevenir teniendo una sola pareja sexual. Estudios similares con adolescentes/jóvenes indican haber recibido información sobre transmisión y prevención del VIH/sida (18); sin embargo, los conocimientos adquiridos no se reflejaban en las prácticas y continuaban presentando ideas erróneas sobre el tema $(8,19)$.

La transmisión del VIH/sida está ligada a los comportamientos sexuales de los adolescentes y jóvenes. En la investigación, el 8,1 \% de la población no ha tenido relaciones sexuales, aspecto que se convierte en un factor protector para este grupo. La mayoria de los estudiantes tiene una vida sexual ción, y algunos estudios $(14,20)$ indican que los estudiantes son conscientes 
de contraer enfermedades de transmisión sexual con dichas prácticas. Otro factor de riesgo encontrado es el inicio temprano de las relaciones sexuales sin protección, y así se evidencia una correlación, esto es, entre mayor edad, mayor uso de condón (7).

Uno de los indicadores de ONUSIDA es identificar las relaciones sexuales antes de los 15 años y el poco uso del condón (21), un factor de riesgo importante de transmisión de VIH/sida en esta población (9,21). El 59,6\% de los encuestados ha tenido más de dos parejas sexuales en el último año, lo que aumenta la vulnerabilidad frente a la infección por VIH/sida $(8,22)$, aspecto que debe tenerse muy en cuenta en el diseño de estrategias educativas y preventivas en los programas de bienestar universitario de las universidades. Aunque en el presente estudio no es muy relevante la población homosexual $(8,9 \%)$, llama la atención comportamientos de riesgo que los exponen a contraer el VIH/sida $(7,23)$.

Llama la atención la similitud de las cifras reportadas entre hombres y mujeres que no utilizaron el condón en su última relación; contrario a otros estudios en los cuales los hombres se exponen más que las mujeres $(22,24)$. El argumento principal para no usarlo es la confianza en la pareja y no verlo como necesario. El uso del condón está relacionado con la percepción que se tiene de la pareja y el tipo de relación $(22,24,25)$. Para muchos, no usar condón o dejar de usarlo con la pareja está asociado a nociones de seguridad, confianza, estabilidad emocional y compromiso con la relación (26). Para otros, la falta de conocimiento y de dinero son razones por las que no se usa (26), si se tiene en cuenta que el presente estudio reporta el lugar que da más confianza o seguridad a la hora de conseguir o comprar condones es la farmacia/droguería (25), seguido del supermercado.

Los jóvenes han tenido relaciones sin condón bajo efectos del alcohol (25,5\%). En Colombia, el consumo de alcohol es socialmente aceptado y se incrementa en los jóvenes cuando se ingresa a la universidad (27-29). Este aspecto es un predictor de conductas sexuales de riesgo $(9,22,29,30)$. El comportamiento es similar entre hombre y mujeres, contrario a otros estudios donde este comportamiento está asociado más a los hombres $(9,28,31)$. Ello hace necesario una política universitaria que refuerce los temas de promoción de la salud, donde involucrar a la familia del universitario es fundamental, y la prevención de situaciones de riesgo en esta población, desde la universidad.

En lo relacionado con la prueba para detectar la infección, los resultados tienen implicaciones importantes para los servicios de salud: baja cobertura en la realización de la prueba en el último año (17,4\%); así como en consejería para la prueba antes $(13,7 \%)$ y después $(11,8 \%)$. Se encontró una relación positiva entre haber oído hablar del VIH/sida y la realización de la prueba, en concordancia con otros estudios, que manifiestan dejar en un segundo plano la consejería para el uso del condón, el sexo seguro y la consejería para la prueba de VIH (25), elementos fundamentales en los programas de salud sexual y reproductiva para adolescentes y jóvenes.

Los estudiantes manifiestan en una baja proporción haber recibido condones en el servicio de salud al que pertenecen (17,6\%). Con mayor frecuencia, los adquieren en las farmacias y menos en los servicios de salud. 
Reportes similares $(25,32)$ indican que uso del condón en la población colombiana es bajo, y es atribuido a la falta de accesos y oportunidad a los servicios de salud en los jóvenes. Esto evidencia las debilidades que tiene el sistema de salud colombiano para la atención de adolescentes y jóvenes, a pesar de contar con una politica de salud sexual y reproductiva.

\section{Conclusiones}

La población estudiada mostró un inicio temprano en sus relaciones sexuales sin protección, un incremento de parejas sexuales en el último año y un uso del condón muy bajo en sus prácticas sexuales. Además, indicó que, a pesar de tener conocimientos sobre el ciclo de la enfermedad y la forma de prevenirla, los jóvenes y adolescentes no tienen en cuenta acciones seguras que los protejan de la infección. Se evidencia la necesidad de hacer un trabajo con acciones orientadas a la promoción de la salud para reducir los riesgos de infección al VIH involucrando las diferentes dependencias de la institución educativa y sectores sociales en la calidad de vida de los universitarios.

El comportamiento ante la transmisión del VIH/sida en adolescentes y jóvenes universitarios en el estudio se tendrá como un referente para orientar acciones de intervención, desde la prevención de la enfermedad y promoción de la salud. También como aspecto para trabajar en las líneas de profundización e investigación en la formación de profesionales en el campo de salud.

\section{Financiación}

Universidad de los Llanos.

\section{Conflictos de interés}

Ninguno declarado.

\section{Agradecimientos}

A las directivas de las universidades participantes, por su consentimiento del estudio; a los adolescentes y jóvenes universitarios, por su participación; a los estudiantes principiantes de investigación del Programa de Enfermería, por su dedicación y responsabilidad; a la Universidad de los Llanos, por el apoyo en la realización del proyecto.

\section{Referencias}

1. Ministerio de la Protección Social-Fondo de Población de las Naciones Unidas. Guía de prevención VIH/sida para jóvenes en contexto de vulnerabilidad [internet]. 2011 [citado 4 jun 2015]. Disponible en: https://www.minsalud.gov.co/salud/Documents/observatorio_vih/ documentos/prevencion/promocion_prevencion/prevencion_poblaciones_vulnerables/a_poblaciones_vulnerables/JOVENES.pdf

2. ONUSIDA. Datos estadísticos mundiales [internet]. 2014 [citado 4 jun 2015]. Disponible en: http://www.unaids.org/es/resources/campaigns/ World-AIDS-Day-Report-2014/factsheet 
3. Universidad CES. Estudio de prevalencia de VIH y comportamientos de riesgo asociados, en usuarios de drogas por vía inyectada (UDI) en la ciudad de Cali-Colombia [internet]. [Citado 4 jun 2015]. Disponible en: http:// www.odc.gov.co/Portals/1/publicaciones/pdf/consumo/estudios/nacionales/CO3832014-informe-estudio-prevalencia-de-vih-cali-diciembre.pdf

4. Ministerio de Salud y Protección Social. Informe GARPR-2014: Seguimiento de la declaración de compromiso sobre el VIH/sida [internet]. 2014. [citado 4 jun 2015]. Disponible en: http://www.unaids.org/ sites/default/files/country/documents//COL_narrative_report_2014.pdf

5. Instituto Nacional de Salud (INAS). Boletín Epidemiológico Semanal [internet]. 2015 [citado 11 ene 2016]. Disponible en: http://www.ins. gov.co/boletin-epidemiologico/Boletn \%20Epidemiolgico/2015\%20 Boletin \%20epidemiologico \%20Semana \%2052.pdf

6. Carratalá E, Espada P, Orgilés M. Conocimientos y actitudes hacia el VIH/sida: diferencias entre adolescentes españoles con padres casados y divorciados. Rev Salud Mental [internet]. 2014 [citado 2 jun 2015]; 36(5):387-91. Disponible en: http://www.inprf-cd.gob.mx/revista_sm/tablas.php?fasciculo=

7. Vélez SV, Serna BB. Los jóvenes: población vulnerable del VIH/sida. Med UPB [internet]. $2011 \mathrm{Feb}$ [citado 22 jun 2015];29(2):144-54. Disponible en: https://revistas.upb.edu.co/index.php/Medicina/article/view/728

8. Rodríguez U, Fernanda A, Vélez V. Susceptibilidad y autoeficacia frente al VIH/sida en adolescentes de Cali-Colombia. Revista Latinoamericana Ciencias Sociales Niñez y Juventud. 2009; 7(2):1513-33.

9. Uribe AF, Orcasita LT. Conductas sexuales de riesgo en estudiantes universitarios de la ciudad de Cali-Colombia. Rev Virtual Univ Católica del Norte [internet]. 2009 [citado 20 jun 2015];27(mayo-agosto):1-31. Disponible en: http://revistavirtual.ucn.edu.co/index.php/ RevistaUCN/article/view/99

10. Intra MV, Roales-Nieto JG, Moreno, SP. Cambio en las conductas de riesgo y salud en estudiantes universitarios argentinos a lo largo del periodo educativo. Int J Psychol Psychol Ther [internet]. 2011 [citado 19 jun 2015];11(1):139-47. Disponible en: http://www.redalyc.org/articulo.oa?id=56017110005

11. Salamanca-Ramos E. Factores asociados a los estilos de vida saludable en estudiantes de los programas de la Facultad de Ciencias de la Salud (FCS) de una Universidad Pública-Villavicencio-Colombia. Referencia. Revista de Enfermagem. 2014;Supl AO2, série IV.

12. García C. Características de la situación de salud en población universitaria. Villavicencio, Colombia: estudio de corte transversal. Rev Colomb Enferm [internet]. 2010 [citado 24 jul 2015];5(5):23-8. Disponible en: http://www.uelbosque.edu.co/sites/default/files/publicaciones/revistas/revista_colombiana_enfermeria/volumen5/caracteristicas_situacion_salud_poblacion_universitaria.pdf

13. Zambrano G, Flórez M, Lozano L, Rubio Y. Prevalencia de los factores de riesgo para cáncer gástrico en universitarios. Cienc y Cuid 
[internet]. 2014 [citado 22 jun 2015];1(1):16-26. Disponible en: http:// revistas.ufps.edu.co/ojs/index.php/cienciaycuidado/article/view/181

14. Morales-Mesa S. Las prácticas sexuales de riesgo al VIH en población universitaria. Rev Salud Pública [internet]. 2014 [citado 22 jun 2015];16(1):27-39. Disponible en: http://www.scielosp.org/pdf/rsap/ v16n1/v16n1a03.pdf

15. Laguado JE, Gómez MP. Estilos de vida saludable en estudiantes de enfermería en la universidad cooperativa de Colombia. Hacia Promoc Salud [internet]. 2014 Jun [citado 26 jun 2015];19(1):68-83. Disponible en: http://www.scielo.org.co/scielo. php?script=sci_arttext\&pid=S0121-75772014000100006\&lng=en

16. Uribe AF, Orcasita LT. Análisis de conocimientos, actitudes, susceptibilidad y auto eficiencia frente al VIH/sida en docentes de instituciones educativas de Cali-Colombia. Rev Psicol Antioquia [internet]. 2011 [citado 26 jun 2015];3(1):39-57. Disponible en: http://pepsic.bvsalud. org/scielo.php?pid=S2145-48922011000100004\&script $=$ sci_arttext

17. Domínguez-Domínguez LE, Cardona-Arias JA. Conocimientos, actitudes y prácticas en adolescentes universitarios entre 15 y 20 años sobre VIH/SIDA en Medellín, Colombia 2013. Medicas UIS [internet]. 2014 [citado 26 jun 2015];27(3):35-45. Disponible en: http://www.sci.unal.edu. co/scielo.php?script=sci_abstract\&pid=S0121-03192014000300005\&ln $\mathrm{g}=\mathrm{es} \& \mathrm{nrm}=$ iso

18. Pineda LTO, Rodríguez AFU, Orbegozo LJV. Knowledge and attitudes toward HIV/AIDS in parents of Colombian adolescents. Rev Colomb Psicol [Internet]. 2013 [citado 28 abril 2016];22(1):59-73. Disponible en: https:// www.scopus.com/inward/record.url?eid=2-s2.0-84880800672\&partn erID $=40 \& \mathrm{md} 5=\mathrm{d} 4 \mathrm{a} 5249 \mathrm{ac} 37 \mathrm{ddd} 0448 \mathrm{~d} 1 \mathrm{a} 85 \mathrm{e} 729 \mathrm{fec} 72$

19. Uribe AF, Orcasita LT, Vergara T. Risk factors in the infection by HIV/ AIDS in Colombian adolescents. Acta Colomb Psicol [internet]. 2010 [citado 26 jun 2015];13(118):11-24. Disponible en: http://www.scielo.org. co/scielo.php?script=sci_arttext\&pid=S0123-91552010000100002\&ln $\mathrm{g}=\mathrm{en} \& \mathrm{tlng}=\mathrm{es}$

20. Morales-Mesa S, Arboleda-Álvarez L, Segura-Cardona A. Las prácticas sexuales de riesgo al VIH en población universitaria. Rev Salud pública [internet]. 2014 [citado 22 diciembre 2015];16(1):27-39. Disponible en: www.scielosp.org/pdf/rsap/v16n1/v16n1a03.pdf

21. ONUSIDA. Presentación de informes sobre los avances en la respuesta mundial al SIDA-2015 [internet]. Ginebra; 2015 [citado 25 jun 2015]. Disponible en: http://www.unaids.org/es/resources/documents/2015/ GARPR_2015_guidelines

22. Arévalo MTV, Sánchez DC, Quintero MA, Ossa HDJZ, Hernández PAH, Cuevas LMT. Prevalencia de prácticas sexuales de riesgo en población adulta de Colombia. Rev Cuba Salud Publica [internet]. 2011 [citado 20 jun 2015];37(4):472-81. Disponible en: http://www.bvs.sld.cu/revistas/spu/vol_37_04_11/spu11411.htm

23. Orcasita L. Palma D, Munévar J. Percepción de riesgo frente al VIH: estudios en mujeres homosexuales. Rev Psicol Univ Antioquia [internet]. 
2013 [citado 26 jun 2015];5(2):63-79. Disponible en: http://aprendeenlinea.udea.edu.co/revistas/index.php/psicologia/article/view/19685

24. Uribe JI, Amador G, Zacarías X, Villarreal L. Percepciones sobre el uso del condón y la sexualidad entre jóvenes. Rev Latinoam Ciencias Soc Ninez y Juv. 2012;10(1):481-94.

25. Valencia CP. Factores que predisponen, facilitan y refuerzan el uso del preservativo en jóvenes universitarios de Cali, Colombia. Rev Salud Pública [internet]. 2012 [citado 21 jun 2015];14(5):810-21. Disponible en: http://www.revistas.unal.edu.co/index.php/revsaludpublica/ article/view/38389/41952

26. Estupiñán-Aponte MR, Amaya-Estupiñán LC, Rojas-Jiménez YA. Representaciones sociales de universitarios sobre la abstinencia sexual y los condones como mecanismos de prevención. Rev Salud Pública [internet]. 2012 Jun [citado 26 jun 2015];14(3):491-501. Disponible en: http://www.scielosp.org/scielo. php?script $=$ sci_arttext\&pid=S0124-00642012000300012\&lng=en.

27. Castaño-Pérez GA, Calderón-Vallejo GA. Problems associated with alcohol consumption by university students. Rev Lat Am Enfermagem [internet]. 2014 [citado 26 junio 2015];22(5):739-46. Disponible en: http://www.scielo.br/scielo.php?script=sci_arttext\&pid=S0104-11692014000500739\&ln $\mathrm{g}=\mathrm{en} \& \mathrm{nrm}=$ iso\&tlng=en

28. Monsalve AS, Espinosa XP, Espinosa NF. Consumo de alcohol en jóvenes universitarios. Av Psicol Latinoam [internet]. 2011 [citado 22 jun 2015];29(1):77-97. Disponible en: http://www.redalyc.org/articulo.oa?id=79920065007

29. Lema, LF. Varela, MT. Bonilla M. Influencia familiar y social en el consumo de alcohol en jóvenes universitarios. Rev Fac Nac Salud Pública [internet]. 2011 [citado 23 jun 2015];29(3):264-71. Disponible en: http://www.redalyc.org/pdf/120/12021452006.pdf

30. Castaño G, Arango E, Morales S, Rodríguez A, Montoya C. Riesgos y consecuencias de las prácticas sexuales en adolescentes bajo los efectos de alcohol y otras drogas. Rev Cubana Pediatr [internet]. 2013 [citado 24 jun 2015];85(1):36-50. Disponible en: http://scielo.sld.cu/ scielo.php?script=sci_arttext\&pid=S0034-75312013000100005\&lng=es

31. González LA, González LV, Botero L. Factores de riesgo relacionados con el estado de salud física de los estudiantes de medicina de una institución en el período 2009-2012. Cienc Salud [internet]. 2014 [citado 25 jun 2015];2(7):11-8. Disponible en: http://revistas.usc.edu.co/ index.php/CienciaySalud/article/view/369

32. Arrivillaga DM, Tovar LM, Correa D. Evidencia poblacional y análisis crítico de determinación social sobre el uso del condón en Colombia. Rev Cuba Salud Pública [internet]. 2012 [citado 26 jun 2015];38(4):553-61. Disponible en: http://bvs.sld.cu/revistas/spu/vol38_4_12/spu07412.htm 
